\title{
A new nonlinear displacement-dependent parametric model of a high-speed rail pantograph hydraulic damper
}

\author{
Wenlin Wang ${ }^{\mathrm{a}, \mathrm{b}, \mathrm{c}^{*}}$, Zirong Zhou ${ }^{\mathrm{a}}$, Weihua Zhang ${ }^{\mathrm{c}}$, Simon Iwnicki ${ }^{\mathrm{d}}$ \\ a School of Mechanical Engineering, Dongguan University of Technology, Dongguan 523808, P. R. of China \\ ${ }^{\mathrm{b}}$ College of Mechanical and Vehicle Engineering, Hunan University, Changsha 410082, P. R. of China \\ c State Key Laboratory of Traction Power, Southwest Jiaotong University, Chengdu 610031, China \\ ${ }^{\mathrm{d}}$ Institute of Railway Research, University of Huddersfield, Huddersfield HD1 3DH, UK
}

\begin{abstract}
A new fully parametric model revealing the nonlinear displacement-dependent characteristics of a high-speed rail pantograph damper has been developed in this study. In the multi-disciplinary physical modeling, the key pressure-flow characteristics of a changeable resistance network and a compression shim-stack valve are formulated, an equivalent-pressure correction factor, $C_{\mathrm{e}}$, is proposed to handle the problem of the shim-stack valve experiencing non-uniform pressure fields, and a finite element analysis (FEA) assisted parameter identification approach of $C_{\mathrm{e}}$ is introduced. Considerable agreement between computer simulation and experiment has validated the damper model. Extensive pantograph-catenary dynamics simulation and experiments were carried out to compare the pantograph dynamic responses when separately using the conventional linear and the new nonlinear damper models, the results show that when designed with the nonlinear damper model, the pantograph would have a softer contact with the catenary when it is raised without prolonging the whole raising time, the operating contact quality of the pantograph and catenary is also significantly improved, and the lowering time of the pantograph is considerably reduced. The new nonlinear damper model is more complete and adaptive to working conditions of the pantograph than the conventional linear damper model, so it is more effective for modern high-speed problem analysis and parameter optimization of the pantograph-catenary system.
\end{abstract}

Keywords: Displacement-dependent; nonlinear damping characteristics; pantograph damper; orifice; parameter identification; pantograph-catenary dynamics

\section{Introduction}

The pantograph plays an important role in current collection by modern high-speed rail vehicles. Optimizing the design of the structural and component parameters of the pantograph will improve

\footnotetext{
* Corresponding author at: School of Mechanical Engineering, Dongguan University of Technology, Dongguan 523808, Guangdong Province, PR China. Tel.: +86 76922861122.

E-mail address: pianowwl@vip.163.com (Wenlin Wang).
} 
pantograph dynamics and enable more stable current collection. A hydraulic damper is usually installed between the base frame and the articulated mechanism (framework) of a pantograph, as illustrated by Figure 1. When the pantograph is lowered, the damper is extended, and when the pantograph is raised, the damper is compressed. The hydraulic damper is a crucial device in improving the pantograph-catenary interaction quality, as well as in obtaining ideal raising and lowering performance of the pantograph.

Figure 1. A simple illustration of the motions of a high-speed rail pantograph and its hydraulic damper.

In previous studies on pantograph-catenary interaction dynamics [1-4] and parameter design optimization of the pantograph-catenary system [5, 6], the pantograph hydraulic damper was all considered a conventional linear model with unidirectional damping, and with the damping coefficient $C$ as the only parameter, as shown in Figure 2(a).

The conventional linear damper model is simple but not adaptive to the changing working conditions of the pantograph. For instance, as the pantograph is raised, the damper has zero damping and an impact will occur when the pantograph begins to contact the catenary; when the pantograph is lowered, a fast descent at the beginning and a soft touch down of the pantograph are expected, but the damper produces the same high-level damping in the whole process; furthermore, when the moving pantograph is contacting the catenary under vibration conditions, the unidirectional and high-level damping characteristics of the damper might not be optimal in pursuing better pantograph-catenary interaction quality.
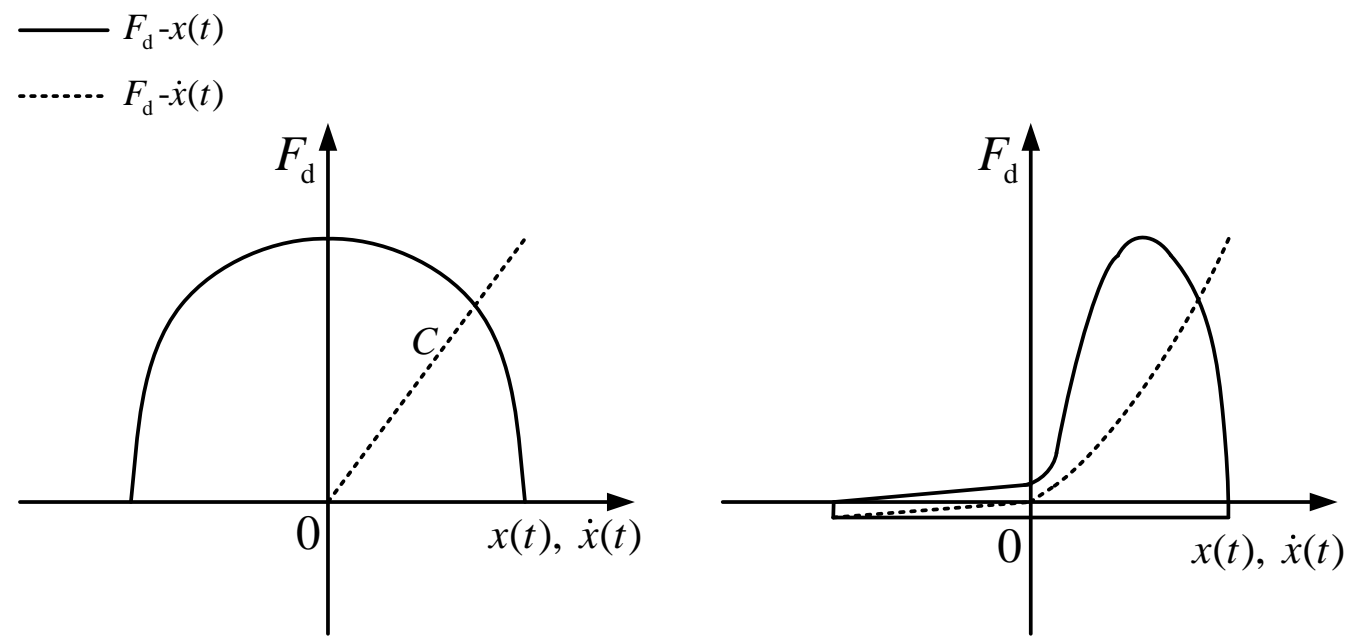
Figure 2. (a) The conventional linear pantograph damper model with unidirectional damping, (b) A modern pantograph damper model with nonlinear displacement-dependent characteristics in its extension stroke and low-level saturation damping characteristics in its compression stroke.

Thus, in modern high-speed pantograph development, a nonlinear damper is usually specified. As shown in Figure 2(b), the damper has nonlinear displacement-dependent characteristics in its extension stroke and low-level saturation damping characteristics in its compression stroke.

Many previous works have been conducted in modeling the damping characteristics of railway hydraulic dampers [7-11] and their influence on rail vehicle dynamics, such as on the yaw motion and stability $[9,12,13]$ and riding comfort [10] of rail vehicle systems. Magnetorheological dampers were also introduced [14] for the semi-active control of rail vehicle secondary suspensions, and other dampers used in the seat suspension of a locomotive driver [15] were also studied. However, research work on parametric modeling of the nonlinear damping characteristics of modern pantograph hydraulic dampers, and the comparison of dynamic responses of the pantograph-catenary system with different damper models, are not widely reported.

In this study, a new parametric physical model describing the nonlinear displacementdependent characteristics of a high-speed rail pantograph hydraulic damper is developed. In the multi-disciplinary modeling, the key pressure-flow characteristics of the displacement-dependent resistance network and the compression valve with an elastic shim-stack are formulated. Parameter identification, simulation and bench testing of the pantograph damper were carried out to validate the new damper model, and a brief comparison of dynamic responses of the pantograph-catenary system with different damper models is given to demonstrate the advantages of the new nonlinear damper model.

The paper is structured as follows: a full mathematical modeling of the nonlinear damper with parameter identification of its compression shim-stack valve is presented in Section 2; Computer simulation, experimental research and model validation of the pantograph hydraulic damper are presented in Section 3; A brief comparison of dynamic responses of the pantograph-catenary system with different damper models are given in Section 4, and concluding remarks are summarized in the last section.

\section{Physical modeling}




\subsection{The principle}

Figure 3 illustrates the structural and working principle of a typical industry high-speed rail pantograph hydraulic damper (Type: J6H36-02-00). In general conditions, the rod-piston assembly of the damper is fixed to the pantograph base frame so that when the pantograph is lowered, the outer tube assembly of the damper is moved by the lower arm mechanism of the pantograph; thus, the damper is extended. In contrast, when the pantograph is raised, the damper is compressed.

During the extension stroke of the damper, as shown by Figure 3(a), the check valve in the piston assembly is closed, while the check valve in the foot valve assembly is opened. Thus, high pressure is built up in the extension chamber, and the high-pressure oil is displaced to the reservoir through the orifice in the inner tube and to the compression chamber through the orifice array in the rod. However, with extension of the damper, the orifices in the rod become sequentially obstructed by the guide seat, causing the pressure in the extension chamber to increase; when all the orifices are shielded, the oil at maximum pressure can only be forced through the orifice in the inner tube. Simultaneously, the oil in the reservoir flows into the compression chamber with very low resistance to compensate for volume expansion of the compression chamber.

Figure 3. Working principles of an industry high-speed rail pantograph hydraulic damper (Type: J6H36-02-00) during its extension stroke (a) and its compression stroke (b).

During the compression stroke of the damper, as shown by Figure 3(b), the check valve in the piston assembly is open, but the check valve in the foot valve assembly is closed. Thus, the extension chamber and the compression chamber unite to become one high-pressure chamber, and the high-pressure oil is displaced to the reservoir through both the orifice in the inner tube and the compression shim-stack valve in the foot valve assembly. During the compression stroke, because pressure in the extension chamber is equal to that in the compression chamber, there is no oil flow in or out of the orifices in the rod.

With the above structural and working principles, the high-speed rail pantograph damper produces nonlinear displacement-dependent damping characteristics, similar to the performance shown in Figure 2(b). However, a traditional linear damper does not have the orifice array in the rod and related displacement-dependent structural designs, so it would produce conventional linear damping characteristics as shown in Figure 2(a). Thus, a high-speed rail pantograph damper would 
be more adaptive to working conditions of the pantograph and perform better in improving the pantograph-catenary interaction quality, as well as in obtaining ideal raising and lowering performance of the pantograph.

\subsection{Multi-disciplinary mathematical modeling}

\subsubsection{Force and fluid continuity equations of the damper}

The instantaneous damping force $F_{\mathrm{d}}(t)$ of the pantograph hydraulic damper can be described as

$$
F_{\mathrm{d}}=-\left\{\frac{\pi}{4}\left(D^{2}-d^{2}\right) P+f_{\mathrm{c}}\right\} \operatorname{sgn}[x(t)]
$$

for the extension stroke, and

$$
F_{\mathrm{d}}=-\left\{\frac{\pi}{4} d^{2} P+f_{\mathrm{c}}\right\} \operatorname{sgn}[x(t)]
$$

for the compression stroke, respectively, where $D, d$ are diameters of the piston and rod, respectively; $P$ is the instantaneous working pressure of the damper; $f_{\mathrm{c}}$ is the total sliding friction force of the moving pairs; and $x(t)$ is the instantaneous displacement of the outer tube assembly.

The fluid continuity equations of the hydraulic damper are written as

$$
\frac{\pi}{4}\left(D^{2}-d^{2}\right)|\dot{x}(t)|=Q_{\text {loss }}+Q_{\text {work }}
$$

for the extension stroke, and

$$
\frac{\pi}{4} d^{2}|\dot{x}(t)|=Q_{\text {loss }}+Q_{\text {work }}
$$

for the compression stroke, respectively, where $Q_{\text {loss }}$ is the instantaneous flow of the total flow loss of the damper and $Q_{\text {work }}$ is the instantaneous flow displaced from the high-pressure chamber.

\subsubsection{Variable oil properties}

The properties of the oil in the pantograph damper vary in relation to the working environment and conditions, and the instantaneous dynamic viscosity $\mu$ and density $\rho$ of the oil can be determined [8] as

$$
\mu=\mu\left(P, T, \varepsilon_{0}\right)=\mu_{0}\left(1+1.5 \varepsilon_{0} \frac{P_{0}}{P}\right) e^{\alpha_{\mathrm{p}}\left(P-P_{0}\right)-\lambda\left(T-T_{0}\right)}
$$

and

$$
\rho=\rho\left(P, T, \varepsilon_{0}\right)=\rho_{0}\left(\frac{1}{1+\varepsilon_{0} P_{0} / P}\right) e^{\beta\left(P-P_{0}\right)-\alpha_{\mathrm{T}}\left(T-T_{0}\right)}
$$


respectively, where $T$ and $T_{0}$ are the instantaneous and reference oil temperatures, respectively; $P_{0}$ is the atmospheric pressure; $\mu_{0}, \rho_{0}, \varepsilon_{0}$ are the dynamic viscosity, density and entrained air ratio of the oil at $P_{0}$ and $T_{0}$, respectively; and $\alpha_{\mathrm{T}}, \beta, \lambda$ are the oil volumetric thermal expansion coefficient, instantaneous oil compressibility coefficient and oil viscosity-temperature coefficient, respectively.

In Equation (6), the instantaneous oil compressibility coefficient $\beta$ is the reciprocal of the volumetric elastic modulus of oil, thus, it can be determined as

$$
\beta=\beta\left(P, \varepsilon_{0}\right)=\left[\frac{1+\varepsilon_{0} \beta_{\mathrm{e} 0}\left(P_{0} / P^{2}\right)}{1+\varepsilon_{0}\left(P_{0} / P\right)}\right] \frac{1}{\beta_{\mathrm{e} 0}}
$$

where $\beta_{\mathrm{e} 0}$ is the elastic modulus of pure oil at $P_{0}$ and $T_{0}$.

\subsubsection{Total flow loss}

Referring to Wang, et al. [8], the flow loss in the pantograph damper due to volumetric oil change can be formulated by

$$
\begin{aligned}
\Delta V_{\text {oil }} & =-\frac{V_{\text {oil }}}{\rho}\left(\frac{\partial \rho}{\partial P} \Delta P+\frac{\partial \rho}{\partial T} \Delta T\right) \\
& =-\frac{V_{\text {oil }} \rho_{0}}{\rho} e^{\beta\left(P-P_{0}\right)-\alpha_{\mathrm{T}}\left(T-T_{0}\right)}\left[\frac{\beta P^{2}+\beta \varepsilon_{0} P_{0} P+\varepsilon_{0} P_{0}}{\left(P+\varepsilon_{0} P_{0}\right)^{2}} \Delta P-\frac{\alpha_{\mathrm{T}} P}{P+\varepsilon_{0} P_{0}} \Delta T\right]
\end{aligned}
$$

where $V_{\text {oil }}$ is the instantaneous volume of the pressure chamber, and for a pantograph hydraulic damper, as shown in Figure 3, $V_{\text {oil }}$ can be determined as

$$
V_{\text {oil }}=\left\{\begin{array}{l}
\frac{\pi}{8}\left(D^{2}-d^{2}\right)\left[H-H_{\mathrm{p}}-2|x(t)|\right], \text { if } \dot{x}(t)<0 \\
\frac{\pi}{8}\left(D^{2}-d^{2}\right)\left[H-H_{\mathrm{p}}+2|x(t)|\right]+\frac{\pi}{8} D^{2}\left[H-H_{\mathrm{p}}-2|x(t)|\right], \text { if } \dot{x}(t)>0
\end{array}\right.
$$

where $H, H_{\mathrm{p}}$ are the heights of inner tube and piston, respectively.

In addition, referring to the approach in Wang, et al. [8] and the concrete structure of the pantograph damper, assuming the back pressure in the reservoir is zero, the flow loss due to pressure leakage can be formulated as

$$
Q_{\text {leak }}=\left\{\begin{array}{l}
\frac{\pi}{6 \mu}\left[\frac{D \delta_{1}^{3}}{2 L}+\frac{d \delta_{2}^{3}}{2 l}+\frac{\delta_{3}^{3}}{\ln \left(R_{2} / R_{1}\right)}\right] P, \text { if } \dot{x}(t)<0 \\
\frac{\pi}{3 \mu}\left[\frac{d \delta_{2}^{3}}{4 l}+\frac{\delta_{3}^{3}}{\ln \left(R_{2} / R_{1}\right)}\right] P, \text { if } \dot{x}(t)>0
\end{array}\right.
$$


where $L, l$ are the seal widths of piston and rod, respectively; $\delta_{1}, \delta_{2}, \delta_{3}$ are the seal or port restriction clearances; and $R_{1}, R_{2}$ are the inner and outer radii of inner tube port restriction, respectively.

Thus, the total flow loss due to volumetric oil change and pressure leakage is summarized as

$$
Q_{\text {loss }}=\Delta V_{\text {oil }}+Q_{\text {leak }}
$$

\subsubsection{Working flow in the extension stroke}

Figure 4(a) is a local cross-section of the rod showing the orifices and hollow passage. There are

six orifices for fluids entering from the extension chamber and five orifices for fluids exiting to the compression chamber, and a hollow passage connects the two groups of orifices. Figure 4(b) illustrates the cross-sections and dimensions of the orifices in the rod.

Figure 5 illustrates the changeable displacement-dependent flow resistance network during the extension stroke of the damper. With extension of the damper, the orifices in the rod become shielded sequentially by the guide seat, so the damping characteristics of the damper vary in relation to its displacement and velocity.

By referring to Figures 4 and 5, the working flow, $Q_{\text {work, }}$ displaced from the high-pressure chamber during the extension stroke of the damper can be formulated by 
$Q_{\text {work }}=\left\{\begin{array}{l}C_{\mathrm{d} 1}\left(\frac{\pi}{4} d_{0}^{2}\right)\left[\frac{2}{\rho}\left(P-P_{\mathrm{i}}\right)\right]^{1 / 2}+C_{\mathrm{d} 1}\left(6 \times \frac{\pi}{4} d_{1}^{2}\right)\left[\frac{2}{\rho}\left(P-P_{\mathrm{i}}\right)\right]^{1 / 2} \\ =C_{\mathrm{d} 1} \frac{\pi}{4}\left[\frac{2}{\rho}\left(P-P_{\mathrm{i}}\right)\right]^{1 / 2}\left(d_{0}^{2}+6 d_{1}^{2}\right), \text { if }-\frac{s_{\mathrm{a}}}{2} \leq x(t)<\left(\frac{s_{\mathrm{a}}}{2}-s_{1}\right), \\ C_{\mathrm{d} 1} \frac{\pi}{4}\left[\frac{2}{\rho}\left(P-P_{\mathrm{i}}\right)\right]^{1 / 2}\left(d_{0}^{2}+5 d_{1}^{2}\right), \text { if }\left(\frac{s_{\mathrm{a}}}{2}-s_{1}\right) \leq x(t)<\left[\left(\frac{s_{\mathrm{a}}}{2}-s_{1}\right)+\Delta s_{1}\right], \\ C_{\mathrm{d} 1} \frac{\pi}{4}\left[\frac{2}{\rho}\left(P-P_{\mathrm{i}}\right)\right]^{1 / 2}\left(d_{0}^{2}+4 d_{1}^{2}\right), \text { if }\left[\left(\frac{s_{\mathrm{a}}}{2}-s_{1}\right)+\Delta s_{1}\right] \leq x(t)<\left[\left(\frac{s_{\mathrm{a}}}{2}-s_{1}\right)+\Delta s_{2}\right], \\ C_{\mathrm{d} 1} \frac{\pi}{4}\left[\frac{2}{\rho}\left(P-P_{\mathrm{i}}\right)\right]^{1 / 2}\left(d_{0}^{2}+3 d_{1}^{2}\right), \text { if }\left[\left(\frac{s_{\mathrm{a}}}{2}-s_{1}\right)+\Delta s_{2}\right] \leq x(t)<\left[\left(\frac{s_{\mathrm{a}}}{2}-s_{1}\right)+\Delta s_{1}+\Delta s_{2}\right], \\ \left.C_{\mathrm{d} 1} \frac{\pi}{4}\left[\frac{2}{\rho}\left(P-P_{\mathrm{i}}\right)\right]^{2}\right), \text { if }\left[\left(\frac{s_{\mathrm{a}}}{2}-s_{1}\right)+\Delta s_{1}+\Delta s_{2}\right] \leq x(t)<\left[\left(\frac{s_{\mathrm{a}}}{2}-s_{1}\right)+2 \Delta s_{2}\right], \\ \left.C_{\mathrm{d} 1} \frac{\pi}{4}\left[\frac{2}{\rho}\left(P-P_{\mathrm{i}}\right)\right]^{2}+d_{1}^{2}\right), \text { if }\left[\left(\frac{s_{\mathrm{a}}}{2}-s_{1}\right)+2 \Delta s_{2}\right] \leq x(t)<\left[\left(\frac{s_{\mathrm{a}}}{2}-s_{1}\right)+\Delta s_{1}+2 \Delta s_{2}\right],\end{array}\right.$

Figure 4. (a) A local cross-section of the rod showing the orifices and hollow passage, (b) an engineering drawing showing cross-sections and dimensions of the orifices in the rod. 


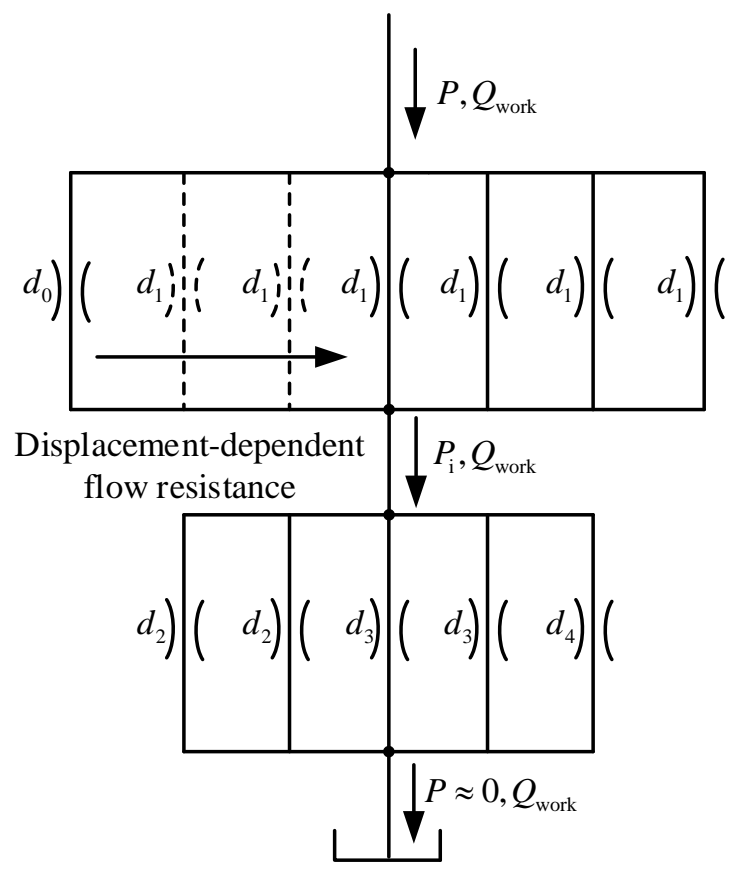

Figure 5. An illustration of the changeable displacement-dependent flow resistance network during the extension stroke of the damper.

where $C_{\mathrm{d} 1}$ is the discharge coefficient of the constant orifice; $d_{0}$ and $d_{1}$ are the diameters of the orifice in the inner tube and that in the rod to permit fluid to enter, respectively; $P_{\mathrm{i}}$ is the pressure in the hollow passage; $s_{\mathrm{a}}$ is the displacement amplitude of the damper; $s_{1}$ is the distance from the first orifice to the point $\left(0, s_{\mathrm{a}} / 2\right)$; and $\Delta s_{1}$ and $\Delta s_{2}$ are the orifice intervals.

If any flow loss in the passage is neglected, according to fluid continuity law, the flow through the passage and that forced out from the orifices in the end of the rod can both be described as

$$
Q_{\text {work }}=C_{\mathrm{d} 1} \frac{\pi}{4}\left(\frac{2}{\rho} P_{\mathrm{i}}\right)^{1 / 2}\left(2 d_{2}^{2}+2 d_{3}^{2}+d_{4}^{2}\right)
$$

where $d_{2}, d_{3}$ and $d_{4}$ are the diameters of the orifices for fluids outflow.

\subsubsection{Working flow in the compression stroke}

\subsubsection{Formulation}

During the compression stroke of the damper, however, when pressure in the extension chamber is equal to that in the compression chamber, theoretically, there would be no oil flow in and out from the orifices in the rod. Thus, if the back pressure in the reservoir is considered to be zero, the working flow in the compression stroke can be expressed as

$$
Q_{\text {work }}=C_{\mathrm{d} 1}\left(\frac{\pi}{4} d_{0}^{2}\right)\left(\frac{2}{\rho} P\right)^{1 / 2}+Q_{\text {valve }}
$$


where $Q_{\text {valve }}$ is the instantaneous flow through the compression shim-stack valve in the foot valve assembly.

According to elasticity mechanics [16], the elastic deflection $w(r)$ of an annular shim clamped at its inner edge and free at its outer edge and subject to a uniform pressure, $P$, can be determined from the following governing differential equation

$$
\left[\frac{d^{2}}{d r^{2}}+\frac{1}{r} \frac{d}{d r}\right]\left[\frac{d w^{2}}{d r^{2}}+\frac{1}{r} \frac{d w}{d r}\right]=\frac{P}{K_{\mathrm{w}}}
$$

where $r$ is the shim radius and $K_{\mathrm{w}}$ is the bending stiffness of the shim and is given by

$$
K_{\mathrm{w}}=\frac{E h^{3}}{12\left(1-v^{2}\right)}
$$

where $E, v$ are the elastic modulus and Poisson's ratio of steel, respectively.

Many classic prior works [17-20] have solved the elastic deflections of shims or shim-stacks for damping valves used in automotive shock absorbers, based on Equation (15) or similar elasticity mechanics differential equations. Wang, et al. [10] deduced the elastic deflection of the shim-stack of a railway hydraulic damper subject to complex non-uniform pressures as

$$
w(r)=\frac{C_{\mathrm{e}} C_{\mathrm{w}} P}{E\left(h_{1}^{3}+h_{2}^{3}+\cdots+h_{\mathrm{n}}^{3}\right)}
$$

where $C_{\mathrm{e}}$ is defined as an equivalent-pressure correction factor; $C_{\mathrm{w}}$ is the deflection coefficient of the shim or shim-stack; and $h_{1}-h_{\mathrm{n}}$ are the thicknesses of the shims in a shim-stack.

Thus, the instantaneous flow forced through the compression shim-stack valve in the foot valve assembly can be formulated by

$$
Q_{\text {valve }}=C_{\mathrm{d} 2}\left[\left.2 \pi r_{\mathrm{s}} w(r)\right|_{r=r_{\mathrm{s}}}\right]\left(\frac{2}{\rho} P\right)^{1 / 2}=C_{\mathrm{d} 2}\left[\left.2 \pi r_{\mathrm{s}} C_{\mathrm{e}} C_{\mathrm{w}}\right|_{r=r_{\mathrm{s}}} \frac{P}{E\left(h_{1}^{3}+h_{2}^{3}+\cdots+h_{\mathrm{n}}^{3}\right)}\right]\left(\frac{2}{\rho} P\right)^{1 / 2}
$$

where $C_{\mathrm{d} 2}$ is the discharge coefficient of the shim-stack valve and $r_{\mathrm{s}}$ is the outer radius of the shim.

\subsubsection{The approach of parameter identification}

The equivalent-pressure correction factor $C_{\mathrm{e}}$ in Equation (18) should be identified before any simulation is performed. Figure 6(a) schematically illustrates the physical meaning of $C_{\mathrm{e}}$. In many cases, a shim or shim-stack in the damping valve of a hydraulic damper is subject to complex non-uniform pressure fields, including both discrete and nonlinear pressure fields, which are different from the uniform pressure field used in Equation (15). In addition, it is both difficult and cost-ineffective to solve the concrete nonlinear laws of the pressures in engineering. Thus, as 
demonstrated by Figure 6(a), assuming there exists a uniform field with pressure $P_{\mathrm{e}}=C_{\mathrm{e}} P$, if the shim-stack deflection under uniform pressure $P_{\mathrm{e}}$ is equal to that under practical nonlinear pressures, then $C_{\mathrm{e}}$ is an equivalent-pressure correction factor.

As an example, the following finite element analysis (FEA) based calculations demonstrate the identification of $C_{\mathrm{e}}$ of the high-speed rail pantograph damper shown in Figure 3 (Type: J6H36-02-00).

The shim-stack of the compression valve in the foot valve assembly of the damper has five identical shims. The shim-stack is subject to a uniform field with pressure $P$ in the annulus from $r_{1}=3.75 \mathrm{~mm}$ to $r_{2}=6.5 \mathrm{~mm}$ and two nonlinear fields, both with pressures decreasing from $P$ to zero; the two nonlinear fields are in the annulus from $r_{1}=3.75 \mathrm{~mm}$ to $r_{0}=2.6 \mathrm{~mm}$ and in that from $r_{2}=6.5$ $\mathrm{mm}$ to $r_{\mathrm{s}}=8 \mathrm{~mm}$.

The maximum shim-stack deflections under real nonlinear pressure conditions were solved by a FEA approach [10], as demonstrated by Figure 6(b), and the results are summarized in Table 1. The theoretical maximum shim-stack deflections under uniform pressure $P$ were also solved and are summarized in Table 1. Thus, the FEA results can be divided by the theoretical results to obtain the equivalent-pressure correction factors. If using the mean value, then $C_{\mathrm{e}}=0.3153$.

In the finite element analysis, because the outer and inner annular areas subjected to nonlinear pressure fields are very small, accuracy is not lost by assuming that the pressure reducing laws are quadratic. In addition, the shim steel elastic modulus $E=2.00 \times 10^{11} \mathrm{~Pa}$, Poisson's ratio $v=3.00 \times 10^{-1}$, and the shim thicknesses $h_{1}=h_{2}=h_{3}=h_{4}=h_{5}=0.5 \mathrm{~mm}$. 


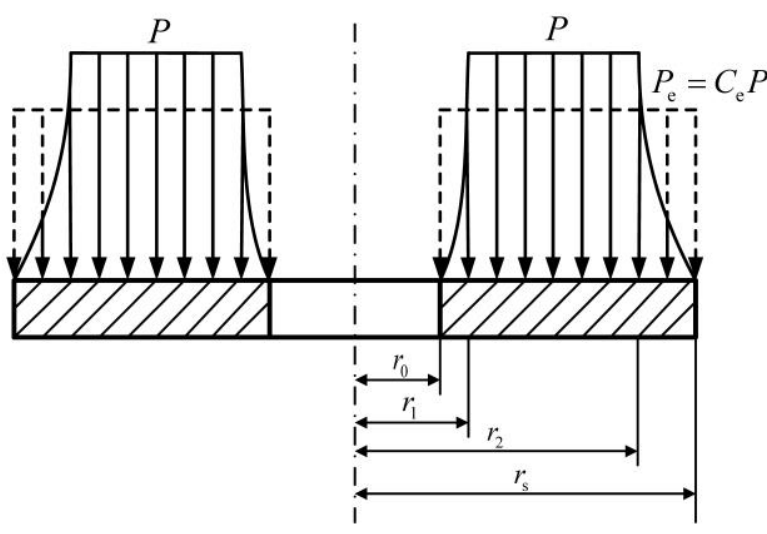

(a)

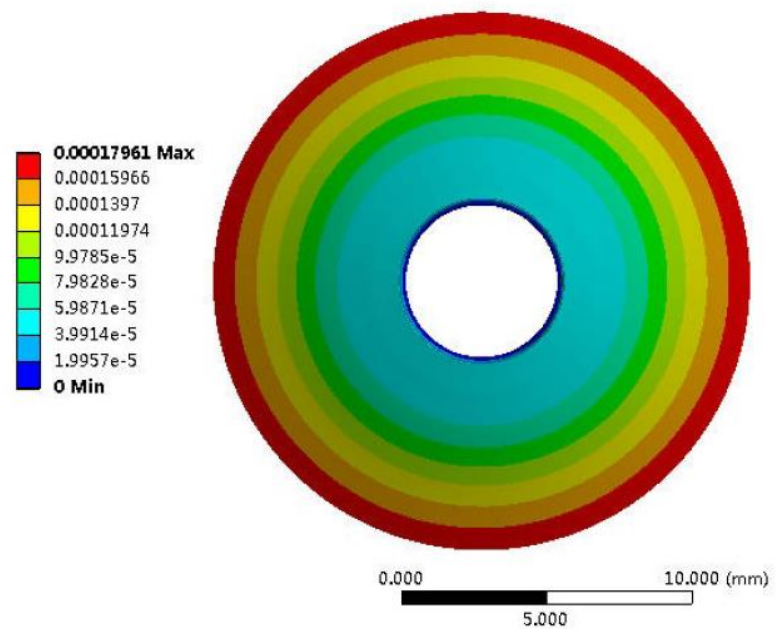

(b)

Figure 6. (a) A schematic illustration of the non-uniform pressure field and physical meaning of the equivalent-pressure correction factor $C_{\mathrm{e}}$, (b) Deflection contour plot of compression shim-stack valve in the foot valve assembly of the pantograph damper (Type: J6H36-02-00) when the shim-stack is subject to a uniform field with pressure $P=1 \mathrm{MPa}$ in the annulus from $r_{1}=3.75 \mathrm{~mm}$ to $r_{2}=6.5 \mathrm{~mm}$ and two nonlinear fields both with pressures decreasing from $1 \mathrm{MPa}$ to zero; the two nonlinear fields are in the annulus from $r_{1}=3.75 \mathrm{~mm}$ to $r_{0}=2.6 \mathrm{~mm}$ and from $r_{2}=6.5 \mathrm{~mm}$ to $r_{\mathrm{s}}=8 \mathrm{~m}$.

Table 1. Maximum shim-stack deflection and equivalent-pressure correction factor of compression valve in the foot valve assembly.

\begin{tabular}{lccccc}
\hline & $\mathbf{0 . 5} \mathbf{~ M P a}$ & $\mathbf{1 ~ M P a}$ & $\mathbf{1 . 5} \mathbf{~ M P a}$ & $\mathbf{2 ~ M P a}$ & $\mathbf{2 . 5} \mathbf{M P a}$ \\
\hline FEA result under real pressure conditions $(\mathrm{mm})$ & 0.000090 & 0.000179 & 0.000269 & 0.000359 & 0.000449 \\
Theoretical result under uniform pressure $P(\mathrm{~mm})$ & 0.000285 & 0.000569 & 0.000854 & 0.001138 & 0.001423 \\
Equivalent-pressure correction factor $C_{\mathrm{e}}$ & 0.3158 & 0.3146 & 0.3149 & 0.3155 & 0.3155 \\
\hline
\end{tabular}

\section{Simulation and experimental validation}

The damping characteristics of a high-speed rail pantograph hydraulic damper (Type: J6H36-02-00) were simulated using the nonlinear displacement-dependent physical model with parameter identification approach introduced in Section 2, and the results are demonstrated by Figures 8. In addition, the key parameters and their values (provided by industry) used in the simulation are shown in Table 2. 
Experimental study of a sample of the pantograph damper was also conducted using the Chinese JS-30 test bench, as shown by Figure 7. The bench test result of the pantograph damper is shown in Figure 8 to compare with the simulation result.

Table 2. Key parameters and their values used in the simulation.

\begin{tabular}{llll}
\hline Parameter & Value & Parameter & Value \\
\hline$C_{\mathrm{d} 1}$ & $7.20 \times 10^{-1}$ & $h_{1}-h_{5}$ & $5.00 \times 10^{-4} \mathrm{~m}$ \\
$C_{\mathrm{d} 2}$ & $6.10 \times 10^{-1}$ & $r_{0}$ & $2.60 \times 10^{-3} \mathrm{~m}$ \\
$D$ & $3.60 \times 10^{-2} \mathrm{~m}$ & $r_{1}$ & $3.75 \times 10^{-3} \mathrm{~m}$ \\
$E$ & $2.00 \times 10^{11} \mathrm{~Pa}$ & $r_{2}$ & $6.50 \times 10^{-3} \mathrm{~m}$ \\
$P_{0}$ & $1.01 \times 10^{5} \mathrm{~Pa}$ & $r_{\mathrm{s}}$ & $8.00 \times 10^{-3} \mathrm{~m}$ \\
$T$ & $4.50 \times 10^{1} \mathrm{C}$ & $s_{\mathrm{a}}$ & $5.00 \times 10^{-2} \mathrm{~m}$ \\
$T_{0}$ & $2.00 \times 10^{1}{ }^{\circ} \mathrm{C}$ & $s_{1}$ & $2.10 \times 10^{-2} \mathrm{~m}$ \\
$d$ & $1.58 \times 10^{-2} \mathrm{~m}$ & $\Delta s_{1}$ & $1.40 \times 10^{-3} \mathrm{~m}$ \\
$d_{0}$ & $6.00 \times 10^{-4} \mathrm{~m}$ & $\Delta s_{2}$ & $3.20 \times 10^{-3} \mathrm{~m}$ \\
$d_{1}$ & $1.10 \times 10^{-3} \mathrm{~m}$ & $\varepsilon_{0}$ & $5.00 \times 10^{-4}$ \\
$d_{2}$ & $1.20 \times 10^{-3} \mathrm{~m}$ & $\mu_{0}$ & $3.81 \times 10^{-2} \mathrm{~Pa} \mathrm{~s}$ \\
$d_{3}$ & $1.10 \times 10^{-3} \mathrm{~m}$ & $v$ & $3.00 \times 10^{-1}$ \\
$d_{4}$ & $1.10 \times 10^{-3} \mathrm{~m}$ & $\rho_{0}$ & $8.75 \times 10^{2} \mathrm{~kg} / \mathrm{m}^{3}$ \\
\hline
\end{tabular}

Figure 7. Experimental study of a high-speed rail pantograph hydraulic damper (Type: J6H36-02-00).

Figure 8(a) compares the tested nominal-speed force vs. displacement $\left(\boldsymbol{F}_{\mathbf{d}}-\boldsymbol{x}(\boldsymbol{t})\right)$ characteristics with the simulated $\boldsymbol{F}_{\mathbf{d}}-\boldsymbol{x}(\boldsymbol{t})$ characteristics of the pantograph hydraulic damper and demonstrates that the test result agrees with the simulation result in a macro sense.

In section "a-b", the damper begins to extend, which means that the pantograph begins to lower. Because all the orifices in the rod are available to charge the fluids in section "a-b", the damping force grows slowly although the excitation speed increases, which is good for the rapid descent of the pantograph. Figure 8(b) is a local view of Figure 8(a) showing the low-level damping force characteristics. Figure 8(b) shows that some differences exist between the test results and simulation results in section "a-b" because of the dynamic hysteresis effect of the fluid resistance network, as shown in Figure 5; the damping force delays at the beginning and leads by the end. However, the normal biases are small and tolerable. 


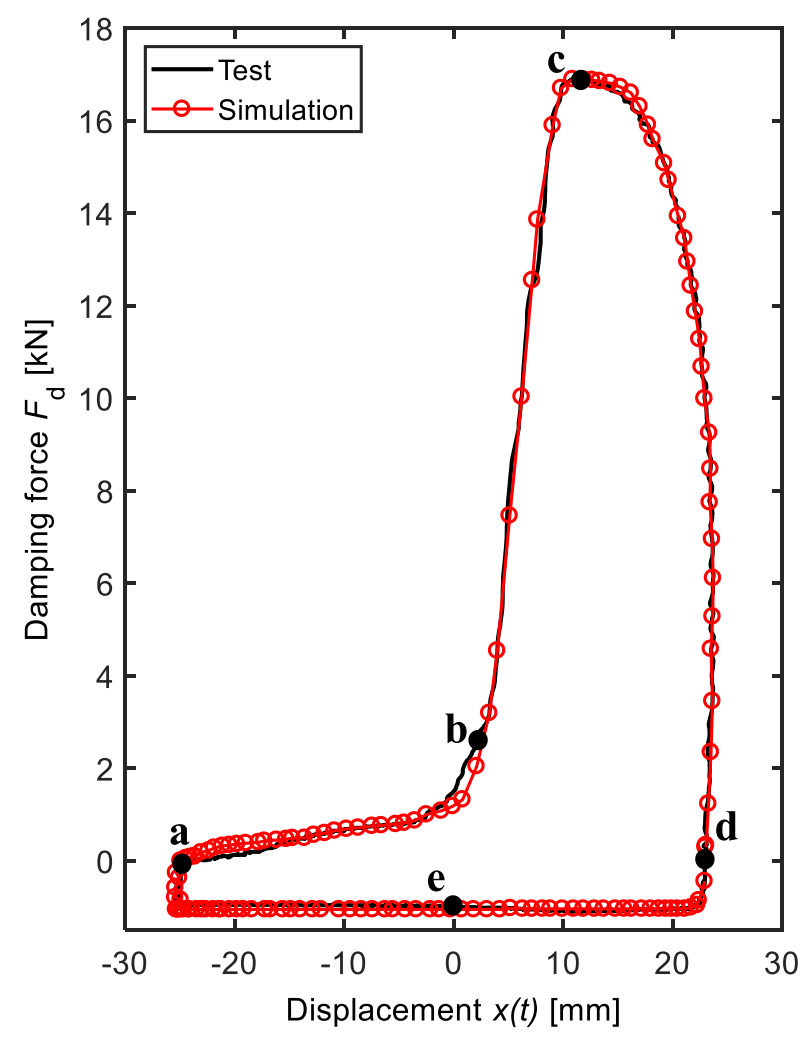

(a)

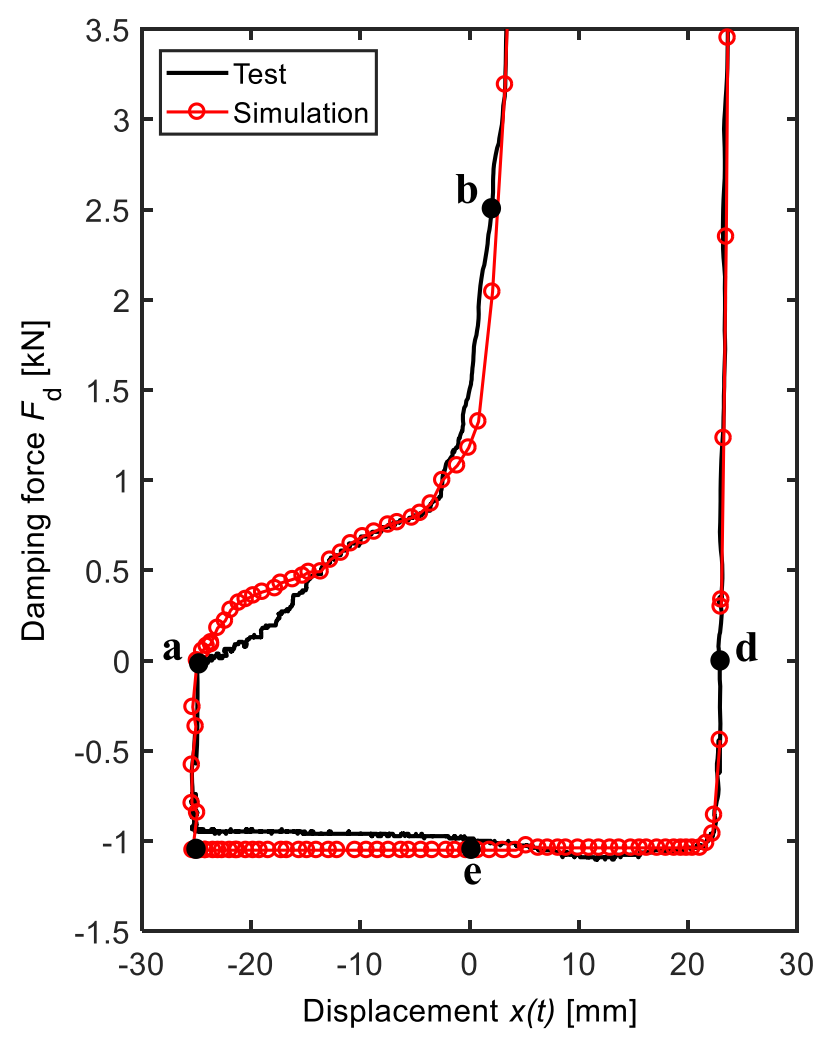

(b)

Figure 8. Nominal-speed force vs. displacement $\left(\boldsymbol{F}_{\mathrm{d}}-\boldsymbol{x}(\boldsymbol{t})\right)$ characteristics (a) and a local view of the $\boldsymbol{F}_{\mathrm{d}} \boldsymbol{x}(\boldsymbol{t})$ characteristics (b) of a high-speed rail pantograph hydraulic damper (Type: J6H36-02-00) with a harmonic excitation of the displacement amplitude of $\pm 24.619 \mathrm{~mm}$, a frequency of $0.64 \mathrm{~Hz}$ and a velocity amplitude of $\pm 0.1 \mathrm{~m} / \mathrm{s}$ (other conditions: $\left.T=45^{\circ} \mathrm{C}, \varepsilon_{0}=0.05 \%\right)$.

In section "b-c", the orifices in the rod begin to be shielded consecutively, so the damping force grows very quickly over a short displacement of $8 \mathrm{~mm}$ or so, and the descent speed of the pantograph rapidly decreases. In section "c-d", only the constant orifice in the inner tube operates, and the pantograph speed is already low and approaching zero, so the damping force descends quickly to zero, and the pantograph is stopped and rests on the vehicle roof. In the key section "b-c-d", the simulation results accurately capture the damping nature of the pantograph hydraulic damper.

In section "d-e-a", the damper is compressed, which means that the pantograph is raised. Because the compression shim-stack valve in the foot valve assembly plays a dominant role and acts as a relief valve in this process, the damper supplies a low-level and approximately constant (saturation) damping force to the pantograph. Figure 8(b) shows that the differences between the test result and simulation result in section "d-e-a" are also small and tolerable. 
Thus, taken together, the established full parametric nonlinear displacement-dependent physical model of a high-speed pantograph hydraulic damper is validated by experimental result, and the model is complete and accurate in all aspects.

\section{A comparison of pantograph dynamic responses when with different damper}

\section{models}

A full mathematical model of the pantograph-catenary system incorporating the new nonlinear displacement-dependent damper model is established, and a thorough simulation of the effect of the nonlinear damping characteristics on the pantograph dynamics is performed and documented in another research paper. However, for paper length and self-containing considerations, this section also gives a brief comparison of the pantograph dynamic responses when separately with the conventional linear and the new nonlinear displacement-dependent damper models.

Figure 9(a) shows the numerical simulation results of the nonlinear displacement-dependent damper model at different excitation speed amplitudes, the damping characteristics are input into the pantograph-catenary dynamics simulation. As a counterpart, a conventional unidirectional damper model with $C=172 \mathrm{kN} \mathrm{s} / \mathrm{m}$, as shown in Figure 2(a), is also input into the simulation. A brief comparison of the pantograph dynamic performance when with different damper models are demonstrated by Figures 10-12.

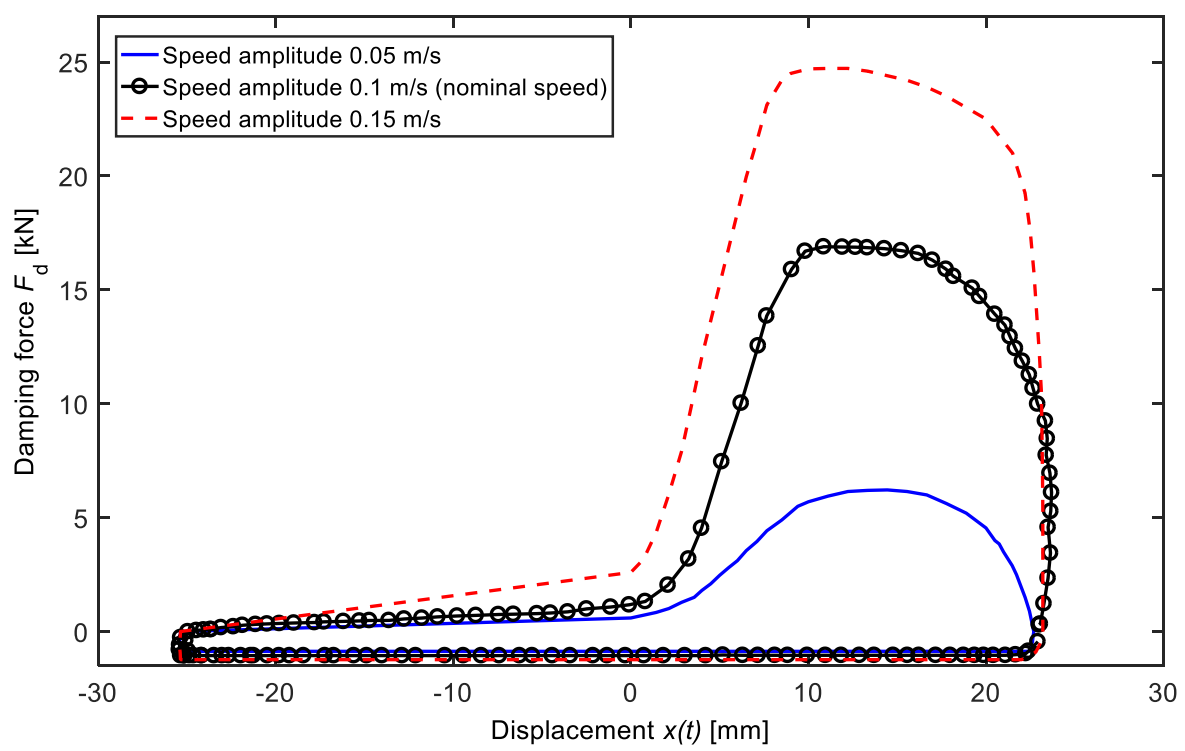

Figure 9. Numerical Simulation results of the nonlinear displacement-dependent damping characteristics at different 
excitation speed amplitudes (Type: J6H36-02-00).

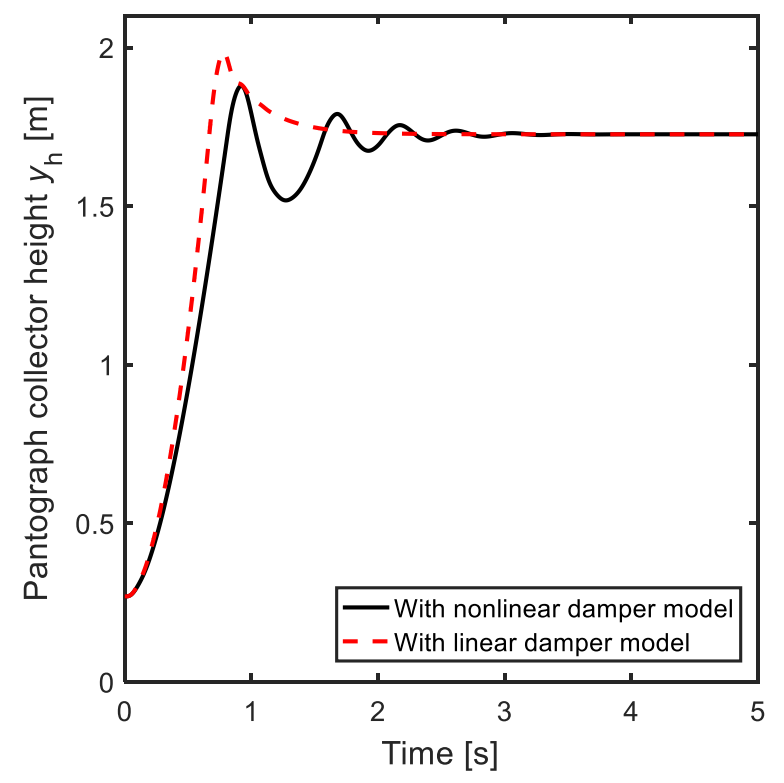

(a)

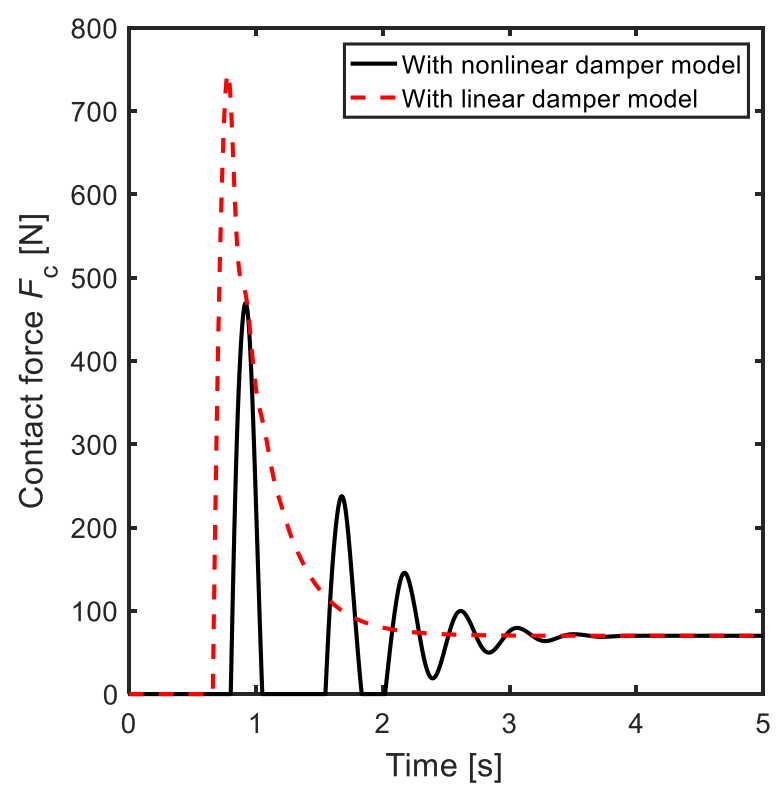

(b)

Figure 10. Raising performance of the pantograph: (a) Instantaneous collector height $y_{\mathrm{h}}$ and (b) contact force $F_{\mathrm{c}}$.

As shown in Figure 10, because there is no damping in the raising process when with the unidirectional linear damper model, the pantograph is quickly raised, and a sharp initial contact (impact) force is also observed (Figure 10(b)) when the pantograph begins to contact with the catenary. The pantograph is stabilized steadily when it goes downward for larger damping coefficient of the linear damper. When with the nonlinear damper, the pantograph is raised in a bit longer time and fluctuates several times when it becomes stabilized, however, the initial contact force is quite smaller than that when with the linear damper. Figure 10 also shows that the difference of pantograph raising time index (stabilization time) is not obvious when with different damper models. 


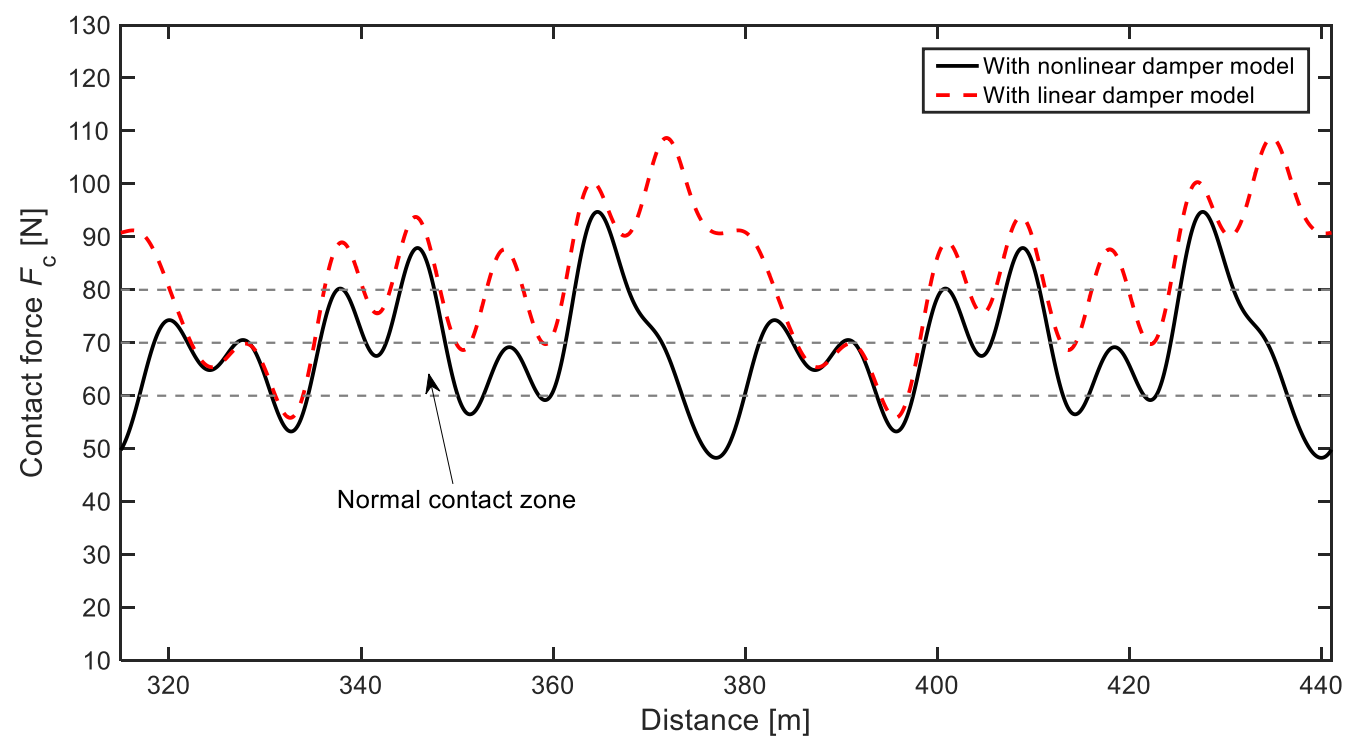

Figure 11. Instantaneous contact force $F_{\mathrm{c}}$ when the pantograph is operating.

Figure 11 compares the instantaneous operating contact forces of the pantograph when with different damper models. When the pantograph is with the nonlinear damper, most of its contact forces fall into the "normal contact zone" [21] and the force peaks are also obviously lower than that when with the linear damper. In other words, the dynamic contact quality of the pantograph and catenary is obviously improved when using a nonlinear displacement-dependent damper.

Figure 12 compares the instantaneous lowering velocities and accelerations of the pantograph when with different damper models. When with the nonlinear damper model, the pantograph has a quick descending at section "a-b" which is related to the small damping section "a-b" of the nonlinear damper in Figure 8(a), then because the pantograph is subject to a large nonlinear damping, the pantograph speed is drastically reduced in section "b-c" and decreases to zero in section "c-d" when the pantograph touches the base frame. However, when with the linear damper model, the pantograph descends slowly and finally touches down due to an unchangeable larger damping coefficient. Figure 12 concludes that the lowering time of the pantograph would be reduced by nearly a half when using a nonlinear damper. 

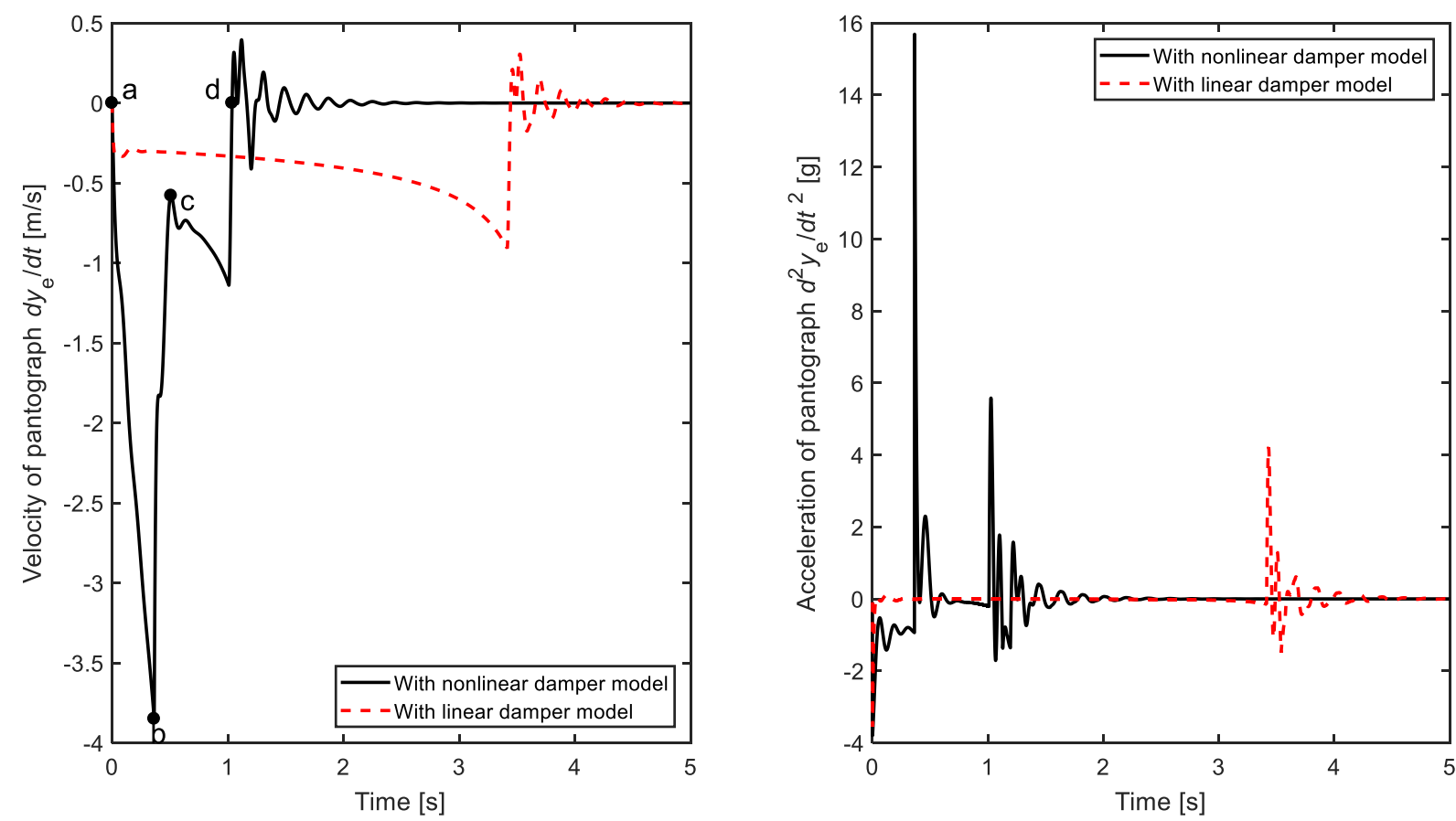

Figure 12. Instantaneous velocity (a) and acceleration (b) of the pantograph (the joint between the upper arm and pan-head suspension) when the pantograph is lowered.

Figure 13 is an experiment rig built in the research work for qualitatively evaluating the pneumatic actuating, raising, lowering and damper performance of the pantograph. The realistic performance of pantograph and damper in many experiments verify that the above numerical simulation results are correct.

Figure 13. An experiment rig for qualitatively evaluating the pneumatic actuating, raising, lowering and damper performance of the pantograph.

\section{Concluding remarks}

(1) A new full parametric model describing the nonlinear displacement-dependent characteristics of a high-speed rail pantograph hydraulic damper has been developed. In the multi-disciplinary physical modeling, the key pressure-flow characteristics of the displacement-dependent resistance network and compression shim-stack valve are formulated, and an equivalent-pressure correction factor, $C_{\mathrm{e}}$, is proposed to handle the problem of the shim-stack 
valve undergoing non-uniform pressure fields, and an FEA-assisted parameter identification approach of $C_{\mathrm{e}}$ is introduced.

(2) Computer simulation and bench testing of a pantograph hydraulic damper were performed. Comparison of the simulation results with the experimental results shows that the parametric physical model accurately captures the damping characteristics, and the new damper model is validated.

(3) Pantograph-catenary dynamics simulation and experiments were carried out to compare the pantograph dynamic responses when separately using the conventional linear and the new nonlinear damper models. The results verify that with the nonlinear damper model, the pantograph would have a softer contact with the catenary when it is raised and without prolonging the whole raising time, the operating contact quality of the pantograph and catenary is also obviously improved, and the lowering time of the pantograph would be reduced to nearly a half of that when using a linear damper model.

(4) The new nonlinear displacement-dependent pantograph damper model is more complete and adaptive to working conditions of the pantograph than the conventional linear damper model, so it is more advantageous and valuable for modern high-speed problem analysis and parameter optimization of the pantograph-catenary system.

\section{Disclosure statement}

No potential conflict of interest was reported by the authors.

\section{Funding}

This work was funded by the National Natural Science Foundation of China (Grant No. 11572123), the Joint Funds of Hunan Provincial Natural Science Foundation and Zhuzhou Science and Technology Bureau (Grant No. 2017JJ4015), the State Key Laboratory of Traction Power in Southwest Jiaotong University (Grant No. TPL1609) and the Research Fund for High-level Talent of Dongguan University of Technology (Project No. GC200906-30).

\section{ORCID}

Wenlin Wang http://orcid.org/0000-0003-2146-6697

Weihua Zhang https://orcid.org/0000-0002-3391-3872

Simon Iwnicki http://orcid.org/0000-0003-1188-7295 


\section{References}

[1] Cho YH. Numerical simulation of the dynamic responses of railway overhead contact lines to a moving pantograph considering a nonlinear dropper. J Sound Vib. 2008; 315:433-454.

[2] Pombo J, Ambrósio J. Influence of pantograph suspension characteristics on the contact quality with the catenary for high speed trains. Comput Struct. 2012;110-111:32-42.

[3] Benet J, Cuartero N, Cuartero F, et al. An advanced 3D-model for the study and simulation of the pantograph catenary system. Transport Res C-Emer. 2013;36: 138-156.

[4] Bautista A, Montesinos J, Pintado P. Dynamic interaction between pantograph and rigid overhead lines using a coupled FEM - multibody procedure. Mech Mach Theory. 2016;97: 100-111.

[5] Zhou N, Zhang WH. Investigation on dynamic performance and parameter optimization design of pantograph and catenary system. Finite Elem Anal Des. 2011;47(3):288-295.

[6] Kim JW, Yu SN. Design variable optimization for pantograph system of high-speed train using robust design technique. Int J Precis Eng Man. 2013;14(2):267-273.

[7] Kasteel RV, Wang CG, Qian LX, et al. A new shock absorber model for use in vehicle dynamics studies. Veh Syst Dyn. 2005;43(9):613-631.

[8] Wang WL, Huang Y, Yang XJ, et al. Nonlinear parametric modeling of a high-speed rail hydraulic yaw damper with series clearance and stiffness. Nonlinear Dyn. 2011;65(1-2):13-34.

[9] Alonso A, Giménez JG, Gomez E. Yaw damper modelling and its influence on railway dynamic stability. Veh Syst Dyn. 2011;49(9):1367-1387.

[10] Wang WL, Zhou ZR, Yu DS, et al. Rail vehicle dynamic response to the nonlinear physical in-service model of its secondary suspension hydraulic dampers. Mech Syst Signal Process. 2017;95: 138-157.

[11] Huang CH, Zeng J. Dynamic behavior of a high-speed train hydraulic yaw damper. Veh Syst Dyn. 2018;56(12):1922-1944.

[12] Mellado AC, Gomez E, Viñolas J. Advances on railway yaw damper characterisation exposed to small displacements. Int J Heavy Veh Syst. 2006;13(4):263-280.

[13] Wang WL, Yu DS, Huang Y, et al. A locomotive's dynamic response to in-service parameter variations of its hydraulic yaw damper. Nonlinear Dyn. 2014;77(4):1485-1502.

[14] Oh JS, Shin YJ, Koo HW, et al. Vibration control of a semi-active railway vehicle suspension with magneto-rheological dampers. Adv Mech Eng. 2016;8(4):1-13.

[15] Stein GJ, Múčka P, Gunston TP. A study of locomotive driver's seat vertical suspension system with adjustable damper. Veh Syst Dyn. 2009;47(3):363-386.

[16] Jiang LP, Tang SG, Wang JM. [Engineering Elasticity Mechanics]. Shanghai(CN): Tongji University Press; 2002. (In Chinese)

[17] Wu YF. [Simulation and design software for automotive shock absorber] [Master's thesis]. Shanghai(CN): Tongji University; 2000. (In Chinese)

[18] Qi HZ, Lei YC, Wu YF, et al. [Precise modelling and simulation of a kind of suspension shock absorber]. Mech 
Sci Tech. 2002;21(5):714-716. (In Chinese)

[19] Li YD, Li J, Song DF, et al. [Research on shim deflection model of automotive shock absorber]. Auto Eng. 2003;25(3):287-290. (In Chinese)

[20] Farjoud A, Ahmadian M, Craft M, et al. Nonlinear modeling and experimental characterization of hydraulic dampers: effects of shim stack and orifice parameters on damper performance. Nonlinear Dyn. 2012;67(2): $1437-1456$

[21] Chinese National Standard GB/T 21561.1-2008: Railway applications - Rolling stock - Pantographs Characteristics and tests - Part 1: Pantographs for mainline vehicles; 2008. (in Chinese) 